\title{
Strategy and Implementation of Multi-mode Control in Switch-Mode Power Supply
}

\author{
Ye Zhao \\ ASIC and System Department \\ Institute of Microelectronics, Chinese Academy of Sciences \\ Beijing, China \\ e-mail: zhaoye@ime.ac.cn
}

\author{
Wei Jiang \\ ASIC and System Department \\ Institute of Microelectronics, Chinese Academy of Sciences \\ Beijing, China \\ e-mail:jiangwei1@ime.ac.cn
}

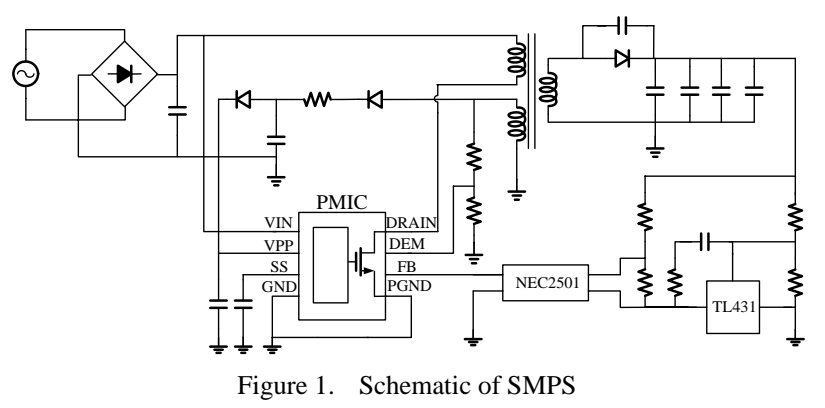

According to Fig. 1, the overall power consumption contains the switching loss, the charge and discharge loss of the gate of the power FET, the conduction loss of the FET, the control circuit loss and other loss. The switching loss PSW and the charge and discharge loss PCH are as follows:

$$
\begin{aligned}
& P_{\mathrm{SW}}=C \cdot f \cdot V_{\mathrm{DS}}{ }^{2} \\
& P_{\mathrm{CH}}=V_{\mathrm{GS}} \cdot Q_{\mathrm{G}} \cdot f
\end{aligned}
$$

The loss of those two types is dominant of the overall power consumption. To curtail the loss, a multi-mode control strategy is presented. In PFM mode, the switching frequency $f$ decreases as the load becomes lighter. In PSM mode, through skipping some pulse cycles, the power FET doesn't switch during these cycles so the loss is avoided. In QR mode, the power FET is on at the valley of $V_{\mathrm{DS}}$ and so the loss $P_{\mathrm{SW}}$ is further decreased.

The conduction loss $P_{\mathrm{CON}}$ is described as:

$$
P_{\mathrm{CON}}=D \cdot I^{2} \cdot R_{\mathrm{DS}(\mathrm{ON})}
$$

In (3), $D$ is the duty radio. The loss can be decreased by limiting the conduct current $I$ to a lower value that can meet the minimum power requirement. $R_{\mathrm{DS}(\mathrm{ON})}$ is related to the technology we adopt and we can decrease it by increase the area properly.

The control circuit loss $P_{\mathrm{CTRL}}$ is:

$$
P_{\mathrm{CTRL}}=V_{\mathrm{PP}} \cdot I_{\mathrm{TOTAL}}
$$

From (4), we can see that lowering $V_{\mathrm{PP}}$ contributes to decreasing the loss. Furthermore, in our design, the chip can work in PSM mode and some circuits shut down to reduce $I_{\text {TOTAL }}$, so the loss is also decreased. 


\section{PROPOSED MULTI-MODE STRATEGY AND CIRCUIT IMPLEMENTATION}

Fig. 2 is the sketch map of multi-mode control. The Yaxis stands for frequency while the $\mathrm{X}$-axis stands for feedback voltage which depends on the load. When the load is heavy, the feedback voltage $\mathrm{V}_{\mathrm{FB}}$ is high and if $\mathrm{V}_{\mathrm{FB}}>\mathrm{V}_{\mathrm{TH} 2}$, the system works in $\mathrm{QR}$ mode. When the load becoming lighter makes $\mathrm{V}_{\mathrm{TH} 1}<\mathrm{V}_{\mathrm{FB}}<\mathrm{V}_{\mathrm{TH} 2}$, the system turns into PFM mode. So, it decreases the switching loss and increase the efficiency of the whole system. To avoid the audible noise issue, a PSM mode is applied that the frequency is clamped at a level about $25 \mathrm{kHz}$ and the system skips some pulses.

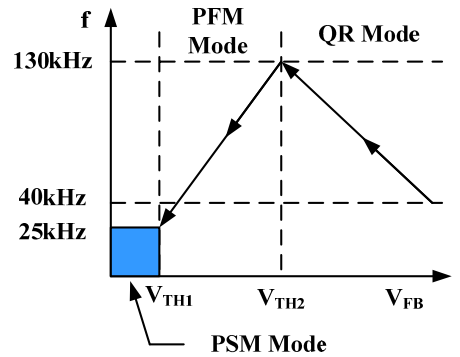

Figure 2. Sketch map of multi-mode control

\section{A. QR mode description}

As we know, there is a conduction resistance $R_{D S}$ in the power FET and when the FET is on, it leads to some conduction loss. The bigger the voltage $\mathrm{V}_{\mathrm{DS}}$ is, the more obvious the loss is. A valley detection circuit is designed to detect the voltage valley of the drain and turns the power FET on at the valley so the conduction loss decreases and the efficiency increases ${ }^{[4]}$. As demonstrated in Fig. 3 (a), the time to turn on the power FET $\mathrm{M}_{0}$ is decided by the valley detection circuit $\mathrm{I}_{4}$.

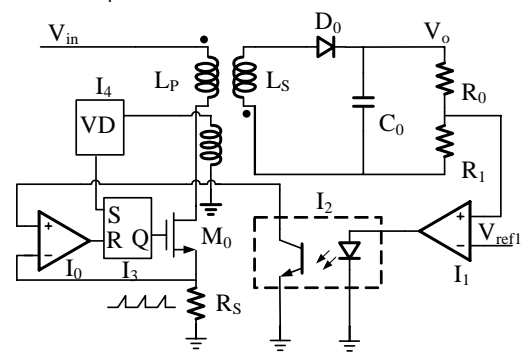

(a)

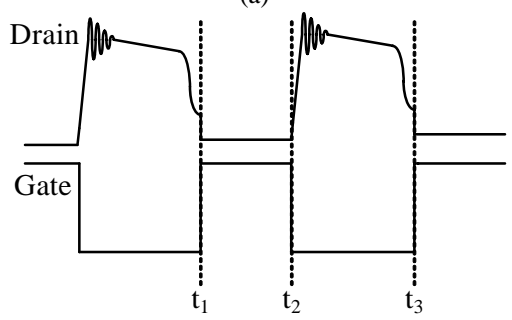

(b)

Figure 3. (a)Schematic of QR mode (b)Waveforms of the drain and gate
Fig. 3 (b) is the waveforms of the drain and gate of the power FET. At the time $t_{1}$, the valley detection circuit detects the voltage valley of the drain and turns the power FET on, so the conduction loss is much smaller. As the FET is on, the voltage on $\mathrm{R}_{\mathrm{S}}$ rises, and when it rises to a threshold at the time $t_{2}$, the power FET is turned off and the drain voltage rises. After a while, the drain begins resonating again and a valley is detected to turn the FET on at the time $t_{3}$ and the cycle repeats.

\section{B. PFM mode description}

In order to solve the problem that the system efficiency is low as the load becomes lighter, the PFM mode is applied. Fig. 4 (a) is the schematic of the PFM mode. In this mode, the on-time of the power FET is fixed and the off-time is variable. The lighter the load is, the longer the off-time is, and so the switching frequency turns lower ${ }^{[5]}$.

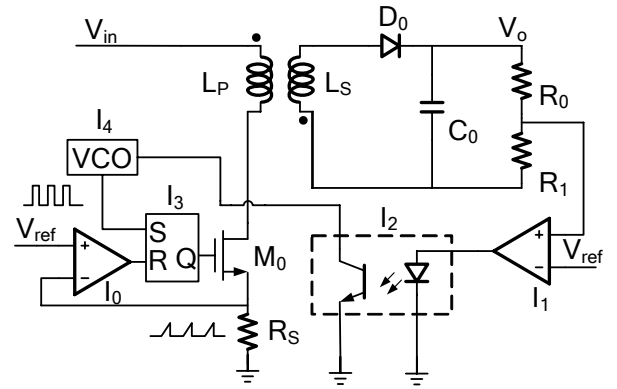

(a)

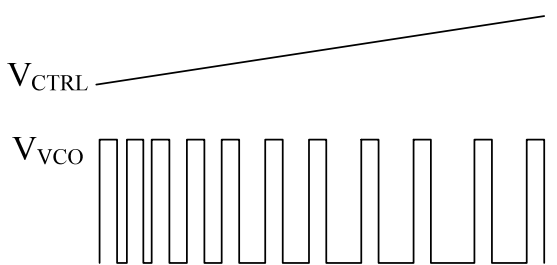

(b)

Figure 4. (a)Schematic of PFM mode (b)Control and output of VCO

In Fig. 4 (b), $\mathrm{V}_{\mathrm{CTRL}}$ is the control signal of the VCO and the $\mathrm{V}_{\mathrm{VCO}}$ is the output. The detailed working process is described as follows: the change of the output voltage,

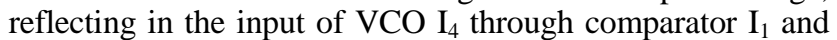
optic coupler $\mathrm{I}_{2}$, causes the frequency of the pulse output of VCO to change. When the load is light, $\mathrm{V}_{\mathrm{CTRL}}$ is high and the frequency is low, and vice versa. The pulse output $\mathrm{V}_{\mathrm{VCO}}$ turns the power FET $M_{0}$ on through RS flip-flop $I_{3}$. As $M_{0}$ is on, the voltage on $R_{S}$ increases linearly, the $V_{\text {in }}$ constant, the rising slope changeless, and $\mathrm{M}_{0}$ turns off as soon as the voltage reaches $V_{\text {ref. }}$ Because the on-time is fixed, the power transferred from $V_{\text {in }}$ to $V_{0}$ is a fixed value. If $V_{0}$ rises, the open frequency of $M_{0}$ falls, so the power transferred to $V_{0}$ decreases and $V_{o}$ falls, otherwise the change reverses.

\section{PSM mode description}

The PSM mode is generally used to regulate the output voltage under either light- or no-load condition ${ }^{[6]}$. Before the output voltage falls to a set point, the power FET and 
some circuits don't work during many cycles, as a result, the power consumption decreases ${ }^{[7]}$.

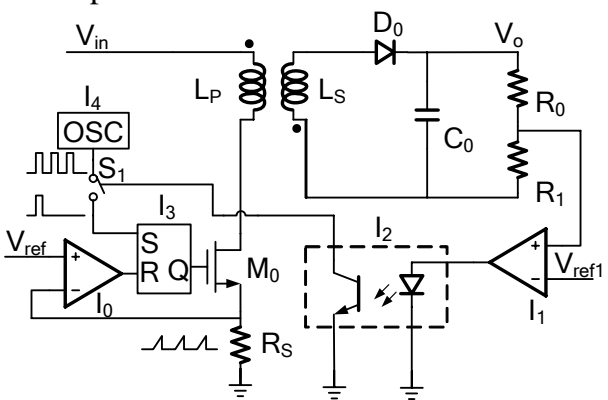

(a)

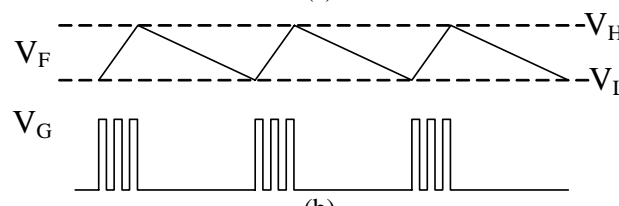

(b)

Figure 5. (a)Schematic of PSM mode (b)Feedback and gate driver signal

Fig. 5 (a) is the schematic of PSM mode, and the feedback signal $V_{F}$ and gate driver signal $V_{G}$ are depicted in Fig. 5 (b). The detailed working process is described as follows: the pulse signal that turns $\mathrm{M}_{0}$ on is produced by oscillator $\mathrm{I}_{4}$ and is in the control of switch $\mathrm{S}$. While $\mathrm{V}_{\mathrm{o}}$ decreases, $V_{F}$ steps down and as soon as it reaches the set point $\mathrm{V}_{\mathrm{L}}$, the switch $\mathrm{S}$ is on, resulting in that the pulses produced by $\mathrm{I}_{4}$ transfer to $\mathrm{S}$ port of $\mathrm{I}_{3}$ and turn $\mathrm{M}_{0}$ on. The cutoff of $\mathrm{M}_{0}$ is controlled by the voltage on $\mathrm{R}_{\mathrm{S}}$. After some cycles, the output voltage rises to the target value and $V_{F}$ reaches the high threshold $\mathrm{V}_{\mathrm{H}}$, then the switch $\mathrm{S}$ turns off and the pulses produced by $I_{4}$ can't transfer to $I_{3}$, so $M_{0}$ is off and $\mathrm{V}_{\mathrm{o}}$ begins to fall down. Accordingly, the feedback signal steps down, so repeat in cycles.

As the efficiency is different with variable load, we integrate all the three modes within one chip. As a result, the efficiency is optimal in the full range of the variable load and the standby power decreases observably.

\section{MEASUREMENT RESULTS}

\section{A. Chip and laboratory prototype}

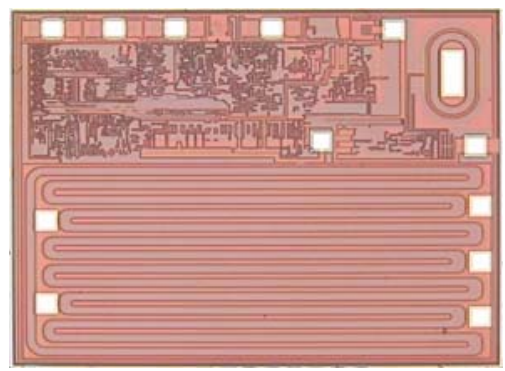

Figure 6. Die photograph of the chip

The proposed multi-mode control circuits are integrated in a chip and the chip is fabricated in the technology of 700V BCD. Fig. 6 is the micro photo of the die and the area is about $2.7 * 2.2 \mathrm{~mm}^{2}$. The chip is encapsulated in the form of DIP-8. As seen in Fig. 7, a laboratory prototype with universal input and $12 \mathrm{~V} / 1 \mathrm{~A}$ output is built with the chip to verify the proposed multimode control strategy. The chip is signed in bold white frame in Fig.7.

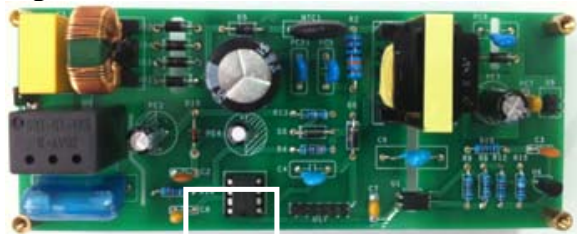

Figure 7. Laborc torv pro otype of SMPS with the chip

\section{B. Measurement results and analysis}

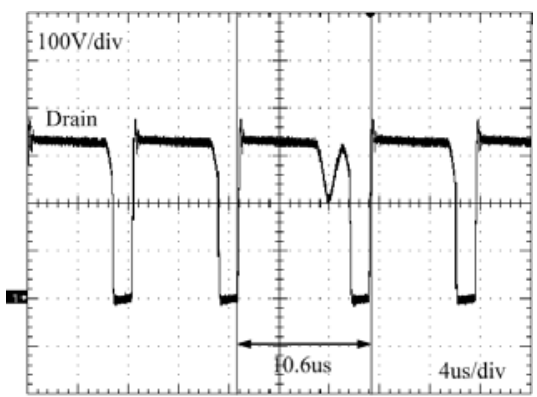

Figure 8. Waveform of drain in QR mode

By connecting a variable load to the output port, we observe the different working modes at different load. From Fig.8 to Fig.10, we can see that the SMPS works in QR, PFM and PSM mode and transmits stably from one mode to another. Fig.8 is the waveform of drain in QR mode, and we can see that the period is 10.6us and the relevant frequency is $94 \mathrm{kHz}$ which is in the designed range of $40 \mathrm{kHz}$ to $130 \mathrm{kHz}$. Fig.9 is the waveform of drain in PFM mode and the frequency is $88 \mathrm{kHz}$, meeting the range of $25 \mathrm{kHz}$ to $130 \mathrm{kHz}$. Fig. 10 is the waveform of gate and $V_{F}$ in PSM mode, and we can see the period is $68 \mathrm{~ms}$ and the frequency is $15 \mathrm{~Hz}$. Such a low frequency ensures the low power consumption in the light- or no-load condition.

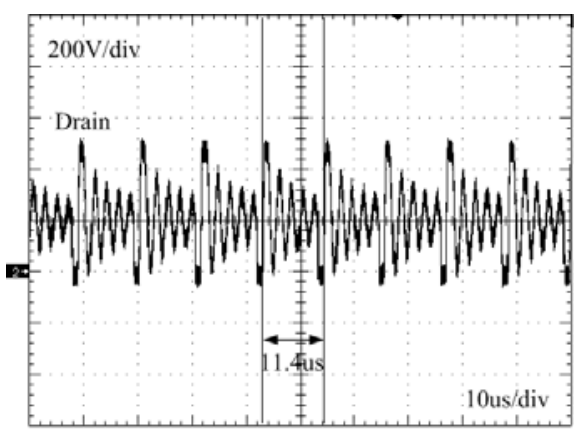

Figure 9. Waveform of drain in PFM mode 


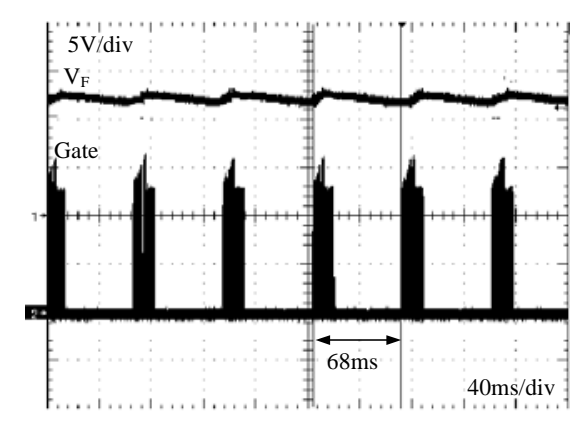

Figure 10. Waveform of gate and $\mathrm{V}_{\mathrm{F}}$ in PSM mode

\section{Performance comparison}

To verify the validity of the proposed strategy, an experiment and test is performed. In the condition of $25 \%$, $50 \%, 75 \%$ and $100 \%$ of the rated output power, we measure the efficiency of proposed multi-mode control strategy and conventional PWM control strategy separately. The efficiency curves of different strategies are depicted in Fig. 11 and from it we can see that the proposed strategy can greatly increase the efficiency compared with the conventional strategy especially when the load is light. Conventional strategy uses constant PWM frequency throughout the entire load range, suffering from poor efficiency in the light-load condition. The test results show that the average efficiency of the proposed SMPS is $84 \%$ and the standby power consumption is $90 \mathrm{~mW}$, which are both better than conventional strategy.

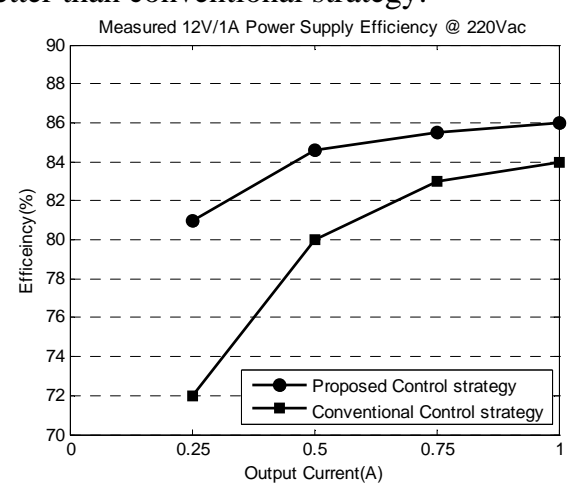

Figure 11. Measured efficiency

\section{CONCLUSIONS}

A low standby power consumption and high efficiency multi-mode control strategy is proposed and a chip that integrates the multi-mode control circuits is fabricated in $700 \mathrm{~V}$ BCD technology. A laboratory prototype is built to verify the validity of the proposed strategy. From the test, we can see that the SMPS can work in QR, PFM and PSM mode and can transmit from one mode to another stably and smoothly. The proposed strategy can reduce the standby power consumption and increase the efficiency in the full range of load. Both the standby power consumption and the average efficiency meet the requirement of latest Energy Star specification with margin.

\section{ACKNOWLEDGMENT}

First and foremost, I would like to show my deepest gratitude to my supervisor, Prof. Zhou Yumei, a respectable, responsible and resourceful scholar, who has provided me with valuable guidance in every stage of the thesis. Her keen and vigorous academic observation enlightens me not only in this thesis but also in my future researching.

I shall extend my thanks to CSMC for all their kindness and help. I would also like to thank Foshan's Industry technology innovation and Incubation Center of CAS who have helped me to develop the fundamental.

Last but not least, I'd like to thank all my friends, especially my team members, for their encouragement and support.

The work was supported by the National Natural Science Foundation of China under Grant 61006058 and a special project on the comprehensive strategic cooperation of Guangdong Province and Chinese Academy of Sciences under Grant 2011A090100010.

\section{REFERENCES}

[1] External AC-DC Power Supplies: Worldwide Forecasts-Tenth Edition, Research and Markets, Dublin, Ireland, April 2011

[2] Savings Estimates for the Report. Lawrence Berkeley National Laboratory, Environmental Energy Technologies Division, http://endues.lbl.gov/info/LBNL-56380(2007).pdf, 2007-3.

[3] ENERGY STAR Program Requirements for Single Voltage Ac-Dc and Ac-Ac Power Supplies, Eligibility Criteria (Version 2.0), 2008, pp.3-5.

[4] Guan-Chun Huang, Tsorng-Juu Liang and Kai-Hui Chen, "Losses Analysis and Low Standby Losses Quasi-Resonant Flyback Converter Design”. IEEE International Symposium on Circuits and Systems, pp.217-220, May 2012.

[5] B.Sahu, G. A. Rincon-Mora, "An accurate, low voltage, CMOS switching power supply with adaptive on-time pulse-frequency modulation (PFM) control”. IEEE Trans. on Circuits and Systems, vol.54 (2), pp.312-318. 2007

[6] D.M.Dwelley. Voltage mode feedback burst mode circuit. US Patent 6307356, Oct.23,2001

[7] SHI Yang, ZHANG Jun-ming, REN Yuan-cheng, Switching mode power supply with burst mode and associated methods of control: US Patent, 8274801. 2012. 